\title{
Interventional Effects of DHA on Behavioral Memory Impairment Caused by Repeated Anesthesia of Sevoflurane in Aged Rats
}

\section{Ming Tian}

Qindao University Medical College Affiliated Yantai Yuhuangding Hospital

Kezhong Li

Qindao University Medical College Affiliated Yantai Yuhuangding Hospital

Xiaoling Zhao ( $\nabla$ xiaoling0125@yeah.net)

Qindao University Medical College Affiliated Yantai Yuhuangding Hospital

Research article

Keywords: DHA, sevoflurane, repeated anesthesia, Nrf2/HO-1 signaling pathway

Posted Date: June 18th, 2020

DOI: https://doi.org/10.21203/rs.3.rs-29872/v1

License: (c) (i) This work is licensed under a Creative Commons Attribution 4.0 International License.

Read Full License 


\section{Abstract}

Backgroud: The current study aimed to explore the effects of Docosahexaenoic acid (DHA) on the behavioral memory impairment induced by repeated anesthesia of sevoflurane in aged rats.

Methods: A total of 45 Sprague-Dawley (SD) aged rats were randomly divided into five groups: Blank control group (Control), sevoflurane group (Model), low-dose DHA group (L-DHA; 0.3g/kg), medium-dose DHA group (M-DHA; $1 \mathrm{~g} / \mathrm{kg}$ ) and high-dose DHA group (H-DNA; $3 \mathrm{~g} / \mathrm{kg}$ ). Morris water maze experiment was used to evaluate the learning and memory ability of rats. Hematoxylin and eosin staining was used to observe histological changes in the hippocampus. Immunohistochemistry and western blot analysis were used to determine the expression of the Nuclear factor erythroid-2 (NF-E2)-related factor 2(Nrf2)/hemeoxygenase-1 (HO-1) signaling pathway.

Results: Rats were indicated to exhibit prolonged escape latency following sevoflurane anesthesia. The number of times taken to cross the platform and the time for target quadrant stay were also demonstrated to be significantly reduced. Rats treated with different doses of DHA were revealed to exhibit reduced escape latency. The number of times taken to cross the platform and the time for target quadrant stay increased. Histopathological examination indicated that DHA attenuated the brain function of the rats that were repeatedly anesthetized using sevoflurane. Furthermore, the expression of Nrf2 and HO-1 protein were demonstrated to be significantly increased.

Conclusions: The present study revealed that DHA has a protective effect on learning and memory impairment in aged rats induced by repeated sevoflurane anesthesia, and the mechanism may be associated with the Nrf2/HO-1 signaling pathway.

\section{Backgroud}

Sevoflurane is a volatile anesthetic that is widely used in clinical anesthesia due to the fact it exhibits high efficiency, is easy to control, exhibits less irritation to the body and has a quick induction and recovery period. However, sevoflurane has potential neurotoxicity and has been previously reported to be associated with postoperative cognitive impairment in elderly patients [1]. In a juvenile animal study, it was demonstrated that sevoflurane mediates neurologic damage and brain dysplasia through oxidative stress [2]. DHA is an unsaturated fatty acid, which is an important component of the neuronal cell membrane [3]. DHA conducts signal transduction between brain function and nerve cells to prevent cytoskeletal protein degradation and the inhibition of oxidative stress and lipid peroxidation. A randomized controlled study revealed a decrease in the incidence of Alzheimer's disease in individuals who included DHA in their diets [4]. The current study explored the effect of different doses of DHA on behavioral memory impairment induced by repeated sevoflurane anesthesia in aged rats, and aimed to provide experimental data for the side effects of repeated sevoflurane anesthesia.

The Nuclear factor erythroid-2-related factor 2/heme oxygenase 1 (Nrf2/HO-1) signaling pathway is a multi-organ protection chain that is induced under a number of different stress environments [5]. The 
Nrf2/HO-1 signaling pathway exerts a variety of different effects, including anti-oxidation, antiinflammatory, reduction of mitochondrial damage, regulation of $\mathrm{Ca}^{2+}$ influx and the regulation of cell death [6-10]. A recent study reported that Nrf2/HO-1 can delay Alzheimer's disease and exert neuroprotective effects [11]. However, there is no clear evidence of its role in repeated anesthesia. Therefore, in the current study, a sevoflurane repeated anesthesia aged rat model was established to determine the role and mechanism of DHA in sevoflurane repeated anesthesia, as well as providing a theoretical basis for exploring the molecular mechanisms of DHA treatment.

\section{Methods}

\section{Experimental animals}

A total of 45 aged SD male rats (Ji'nan Peng Yue Experimental Animal Breeding Co., Ltd.; production

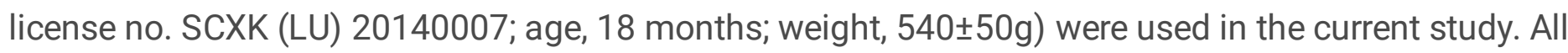
animals were quarantined as required. During the quarantine period, animal activities, including diet, were observed. Animals were required to pass quarantine prior to participation in the subsequent experiment. The environmental conditions for laboratory animal feeding and management were as follows: Room temperature $20-26^{\circ} \mathrm{C}$, daily temperature difference $\leq 4^{\circ} \mathrm{C}$, relative humidity $40-70 \%$, alternation between light and dark $12 / 12 \mathrm{~h}$. During the quarantine and experimental period, the rats had access to food and water ad libitum. During housing, animals were monitored twice daily for health status. No adverse events were observed. All experimental protocols were conducted according to the Nursing and Use Guidance for Animal Experiment Operation guidelines of the National Institutes of Health (NIH Pub. no. 85-23; revised in 1996). and were approved by the Animal Protection and Use Committee of the Affiliated Yantai Yuhuangding Hospital of Qingdao University. All sections of this report adhere to the ARRIVE Guidelines for reporting animal research [12]. A completed ARRIVE guidelines checklist is included in Checklist S1.

\section{Animal grouping, anesthesia and administration}

A total of 45 sprague dawley rats were randomly divided into 5 groups: Blank control group (Control), sevoflurane (NMPN-H20070172; Shanghai Hengrui Pharmaceutical Co., Ltd.) group (Model), low-dose DHA group (Rongcheng Baihe Biotechnology Co., Ltd.; L-DHA; $0.3 \mathrm{~g} / \mathrm{kg}$ ), medium-dose DHA group (MDHA; $1 \mathrm{~g} / \mathrm{kg}$ ) and high-dose DHA group (H-DHA; $3 \mathrm{~g} / \mathrm{kg})$, with 9 rats in each group.

Rats in the Model, L-DHA, M-DHA and H-DHA groups were placed in a self-made transparent anesthesia box $(50 \times 40 \times 40 \mathrm{~cm})$ from days 11 to day 15 of the experiment. One side hole of the box was connected to a Drager anesthesia machine (Drager Company, Germany), which administered the rats' with $2.5 \%$ sevoflurane anesthetic, meanwhile the heart rate, respiratory frequency and blood oxygen saturation were continuously monitored using an electrocardiogram monitor (NORDEP LTD) to eliminate brain damage caused by hypoxia. When the rats were induced into a coma, they were immediately exposed to air to wake them. After their righting reflex was recovered and they were able to move freely, the rats were then 
placed in the box again for another dose of anesthetic. This procedure was repeated 5 times per day for 5 days. The control group received no treatment.

Rats in the L-DHA, M-DHA and H-DHA groups received daily intragastric administration of DHA on days 110 of the experiment (10 days prior to the administration of sevoflurane), and received 5 days of DHA administration and daily inhalation of sevoflurane for repeated anesthesia, while the Control and Model group received no treatment.

Rats in the Model, L-DHA, M-DHA and H-DHA groups received sevoflurane inhalation for anesthesia prior to the Morris water maze test from days 16-20, and no drugs was administered until day 21 of the test. Sevoflurane anesthetized rats were trained 20 min after the righting reflexes were restored.

\section{Morris water maze experiment}

\section{Learning capability test (positioning navigation experiment)}

Rat navigational abilities were assessed using Morris water maze experiments. The Morris Water Maze (MWM) (Institute of Materia Medica; Chinese Academy of Sciences) consisted of two parts: One round pool (120 cm in diameter; $50 \mathrm{~cm}$ in height; stainless steel) and one movable platform. The platform was located $2 \mathrm{~cm}$ below the water. The water temperature of the pool was $\sim 25^{\circ} \mathrm{C}$. The pools surface was divided into four parts and was set as four quadrants (quadrants 1,2,3 and 4). Within a specified period of time or when the rats begin to swim until they climbed onto the platform, the computer automatically tracked the rats' swimming trajectories and calculated the incubation period (the time when the rats located the platform in the pool) automatically using software (Etho Vision XT, Noldus Information Technology BV). The positioning navigation experiment recorded the time taken for the rats to locate the platform hidden under the water surface, and tested the spatial orientation learning ability of the rats.

Rats entered the swimming test from the center of any quadrant facing the pool wall. If the animal failed to locate the platform in the pool or climbed up the platform within $120 \mathrm{sec}$, then time taken was calculated as $120 \mathrm{sec}$. The rat was then guided and placed on the platform for $30 \mathrm{sec}$. The rats were subsequently removed from the platform and wiped dry. After the rats rested for $60 \mathrm{sec}$, they were trained for the next round. Rats were trained four times a day for five consecutive days. The average of the rats' four training incubation periods was taken as the rat's daily learning achievement.

\section{Memory test (space exploration experiment)}

A period of $24 \mathrm{~h}$ after the end of the positioning navigation experiment, and on day 21 of the experiment, the platform was withdrawn and the rats were placed in water at any of the same points of entry. The point of entry was the same as the position in the positioning navigation experiment. The rats were allowed to swim for $120 \mathrm{sec}$, and the time of target quadrant stay and the number of attempts to cross the platform were recorded.

\section{HE staining}


After the rats' behavioral tests were completed, all animals were sacrificed by cervical spine dislocation after anesthetized by intraperitoneal injection of $1 \%$ pentobarbital sodium $40 \mathrm{mg} / \mathrm{kg}$. Brain tissues were dissected out and placed in an ice bath, with the hippocampus being isolated from the brain.

Histopathological examination was then performed using formalin fixation. The samples were embedded in paraffin and cut into $4 \mu \mathrm{m}$ sections. The paraffin sections were dewaxed using xylene, dehydrated with serial dilutions of ethanol, stained with hematoxylin and eosin (H\&E) (Beijing Solarbio Science \& Technology Co., Ltd.), and rinsed in distilled water for $30 \mathrm{sec}$. The sections were washed with $95 \%$ anhydrous ethanol, soaked twice for $1 \mathrm{~min}$, placed in a xylene soak three times for $5 \mathrm{~min}$, and permount mounting medium (Thermo Fisher Scientific, Inc.) was used to place the samples on a coverslip, and pathological changes were observed using an optical microscope (magnification, x400; Olympus Corporation).

\section{Immunohistochemistry}

The hippocampal tissues were embedded in paraffin and sectioned $(5 \mu \mathrm{m})$. The sections were deparaffinized with xylene twice, and rehydrated in a descending ethanol series. Endogenous peroxidase was inhibited by incubating the sections for 30 min using $3 \% \mathrm{H}_{2} \mathrm{O}_{2}$. Antigen retrieval was performed using a citrate buffer at a high temperature for $10 \mathrm{~min}$. The sections were subsequently blocked for 20 min in 5\% BSA, and incubated using a primary anti-HO-1 antibody (1:100; cat. no. ab13243; Abcam), and an anti-Nrf2 antibody (1:100; cat. no. ab31163; Abcam) overnight at $4^{\circ} \mathrm{C}$. After rewarming, sections were incubated with a horseradish peroxidase (HRP)-conjugated goat anti-rabbit IgG secondary antibody (1:1000; cat. no. ab6721; Abcam) at $37^{\circ} \mathrm{C}$ for $1 \mathrm{~h}$. Sections were visualized with 3, 3-diaminobenzidine tetrahydrochloride (DAB) as the chromogen (Beijing Solarbio Science \& Technology Co., Ltd.). The sections were dehydrated and placed onto a coverslip with Permount mounting medium (Thermo Fisher Scientific, Inc.). The slides were observed under an optical microscope (Olympus Corporation; magnification, $\mathrm{x} 200$ ). The data were expressed as the percentage of positive cells out of the total number of cells counted.

\section{Western blot analysis}

Total protein of the hippocampal tissues was extracted using RIPA lysis buffer containing proteinase inhibitor cocktail, and the concentration of protein was determined using a BCA assay. Proteins $(50 \mu \mathrm{g})$ were separated using 10\% SDS-PAGE and transferred onto PVDF membranes (EMD Millipore). The membranes were blocked using $5 \%$ skim milk at $4^{\circ} \mathrm{C}$ overnight, then incubated with primary anti Nrf2 antibody (1:1,000; cat. no. ab137550; Abcam) and anti HO-1 antibody (1:2,000; cat. no. ab13243, Abcam) at $4^{\circ} \mathrm{C}$ overnight. After incubating with secondary antibody sheep anti-rabbit IgG (1:5,000; cat. no. ab97095; Abcam) at $37^{\circ} \mathrm{C}$ for $1 \mathrm{~h}$, protein bands were visualized using the ECL chemiluminescence system (Thermo Fisher Scientific, Inc.). Protein expression levels were normalized to $\beta$-actin (1:2,000; cat. no. ab8227; Abcam) and quantified using Image $\mathrm{J}$ software version 1.46 (National Institutes of Health).

\section{Statistical analysis}


All experimental data are expressed as mean \pm standard deviation, and processed using SPSS 20.0 (IBM Corp.). Multiple comparisons were evaluated using a repeated measures ANOVA. A one-way analysis of variance (ANOVA) was used to compare the mean of multiple groups with a Dunnett's post-hoc test. $\mathrm{P}<0.05$ was considered to indicate a statistically significant difference.

\section{Results}

\section{Rat learning ability test}

Compared with the Control group, the escape latency of rats in the Model group was demonstrated to be significantly increased after inhalation of sevoflurane $(P<0.05)$. Compared with the Model group, the escape latency of rats in the L-DHA, M-DHA and H-DHA group were decreased, exhibited a dosedependence on DHA, and the H-DHA group was indicated to be significantly different $(P<0.05$; Table I).

\section{Rat memory ability test situation}

As presented in Fig. 1, compared with the Control group, the number of times to cross the platform and the time for target quadrant stay of rats in the Model group was significantly increased after inhalation of sevoflurane $(P<0.05)$. Compared with the Model group, the number of times to cross the platform and the time for target quadrant stay of rats in the L-DHA, M-DHA and H-DHA groups were increased, and the HDHA group was indicated to exhibit a significant difference $(P<0.05)$.

\section{Histopathological examination of the rats' hippocampus}

As presented in Fig. 2, the hippocampus in the control group was normal (CA1) except for occasional vasodilatation, while the Model, L-DHA, M-DHA and H-DHA group all exhibited pathological change to some extent in the CA1 area (Fig. 2). Pathological changes, including the disordered arrangement of neurons, deep staining of neuronal nucleus pyknosis, cell edema and microglia foaming were observed in the Model group, and some areas were indicated to exhibit a small amount of cell necrosis. The pathological changes in the L-DHA and M-DHA groups were slightly reduced compared with those in the Model group, and neurons exhibited a disordered arrangement, along with pyknosis and deep staining to neuronal nucleus, and cell edema was observed using a microscope. Pathological changes in the H-DHA group was least obvious, and included neuron disorder arrangement.

\section{Changes in Nrf2 and HO-1 protein}

The results of immunohistochemistry demonstrated that the positive expression of Nrf2 and HO-1 was mainly located in the hippocampus and cortex of the brain. The positive expression in the control group was not obvious. The positive expression in the L-DHA, M-DHA and H-DHA groups were gradually increased. The H-DHA group was significantly increased compared with the model group $(P<0.01 ;$ Fig. 3$)$. The results were consistent with the results of western blot analysis. 
Compared with the control group, the expression of Nrf2 and HO-1 protein in the model group was increased ( $\mathrm{P}<0.05$; Fig. 4), and the expression of Nrf2 and HO-1 protein in the L-DHA, M-DHA and H-DHA groups were more obvious. However, in the H-DHA group, the Nrf2 and HO-1 protein was significantly increased $(P<0.01)$. The results indicated that DHA may decrease the damage of repeated anesthesia on the brain tissue of rats, and this mechanism may be associated with Nrf2 and HO-1 protein levels.

\section{Discussion}

A study has confirmed that long-term exposure to sevoflurane can lead to neurodevelopmental disorders and impaired learning and memory in juvenile animals [11]. In addition, clinical studies in elderly patients have also indicated that exposure to sevoflurane is associated with postoperative cognitive function [13]. The neurotoxicity of sevoflurane is time-and dose-dependent [14]. A study hase demonstrated that exposure to sevoflurane in pregnant rats can lead to brain damage in newborns within two weeks of birth [15]. Furthermore, behavioral experiments in grown rats have also indicated that the learning and memory abilities of the sevoflurane exposed group were lower compared with the control group. In laboratory animals exposed to sevoflurane during the peak period of neurodevelopment, synaptic plasticity and long-term potentiation were affected, and learning and memory ability was decreased $[16,17]$. In the current study, after rats were exposed to repeated sevoflurane anesthesia, they were observed to exhibit aimless movement, no effective search of the platform, a longer space exploration period and decreased time taken to cross the platform, which was consistent with the aforementioned studies.

DHA, which is one of the substances that forms the normal structure of the brain and is easily obtained from deep-sea fish oil, is often processed as a health product or a nutritional supplement to be added to food. A study has indicated that the incidence of neurodegenerative diseases is lower in populations that adopt a mediterranean diet, which may be related to the high content of DHA and other substances during long-term intake [18]. In studies assessing elderly rodents, DHA and/or eicosapentaenoic acid supplements have been indicated to improve cognitive performance and induce protection against neuroinflammation and oxidative stress $[19,20]$. In the present study, after different doses of DHA were provided to aged rats with repeated sevoflurane anesthesia, the spatial exploration ability and navigation ability were attenuated in a dose-dependent manner, indicating that DHA could effectively intervene and treat the spatial learning and memory impairment induced by repeated sevoflurane anesthesia. The histopathological examination results of the hippocampus in rats also suggested that DHA could improve brain function, which was damaged by repeated sevoflurane anesthesia, and alleviated cognitive impairment. Moreover, with an increasing dose of DHA, the intervention effect was enhanced in a dosedependent manner. The results of the current study suggested that DHA had a protective effect on learning and memory impairment induced by repeated sevoflurane anesthesia in aged rats.

The activation of Nrf2/HO-1 is believed to serve an important role in the development of brain injury [21]. The Nrf2/HO-1 pathway serves a crucial role in the body's anti-oxidative stress response, and Nrf2 nuclear translocation is the key to HO-1 activation [22]. In the current study, immunohistochemistry and western blot analysis were used to verify whether DHA induced anti-oxidative stress via the Nrf2/HO-1 pathway. 
The results demonstrated that the expression of Nrf2 and HO-1 proteins were significantly increased in each dose group of DHA compared with the model group, and this effect was dose-dependent.

\section{Conclusions}

In conclusion, the results of the present study indicated that DHA exhibited a protective effect on learning and memory impairment in aged rats, which was caused by repeated sevoflurane anesthesia. The increasing expression of Nrf2 and HO- 1 indicated that the mechanism may associated with the Nrf2/HO1 signaling pathway.

\section{Abbreviations}

DHA: Docosahexaenoic acid

Nrf2: Nuclear factor erythroid-2-related factor 2

HO-1: heme oxygenase 1

BSA: bovine serum albumin

DAB: 3, 3-diaminobenzidine tetrahydrochloride

SDS-PAGE: polyacrylamide gel electrophoresis

PVDF: polyvinylidene fluoride

\section{Declarations}

\section{Ethics approval and consent to participate}

The present study was approved by Ethics Committee of the Affiliated Yantai Yuhuangding Hospital of Qingdao University (YHDQD2055).

\section{Consent for publication}

Not applicable.

\section{Availability of data and materials}

The datasets generated and/or analyzed during the current study are available from the corresponding author on reasonable request.

\section{Competing interests}

There is no conflict of interest in this study. 


\section{Funding}

This research was supported by Science and Technology Project of Yantai City (Project No.: 2016WS009). The funding provided experimental equipment and implementation for the study.

\section{Author's contributions}

TM, LK and ZX designed the study. TM and LK performed experiments. TM, LK and ZX performed data analysis, interpreted the data and acquired samples. TM and LK contributed pathological analysis. TM and ZX wrote the manuscript. All authors read and approved the final manuscript.

\section{Acknowledgments}

Not applicable.

\section{References}

1. Gross AF, Stern TA. Neuropsychiatric conditions associated withanesthesia exposure. Psychosomatics. 2014; 55(1): 21-28.

2. Yufune S, Satoh Y, Akai R, Yoshinaga Y, Kobayashi Y, Endo S, Kazama T. Suppression of ERK phosphor rylation through oxidative stress is involved in the mechanism under lying sevofluraneinduced toxicity in the developing brain. Sci Rep. 2016; 6: 21859.

3. Kabuto H, Amakawa M, Mankura M, Yamanushi TT, Mori A. Docosahexaenoic acid ethyl ester enhances 6-hydroxydopamine-induced neuronal damage by induction of lipid peroxidation in mouse striatum. Neurochem Res. 2009; 34(7): 1299-1303.

4. Hartmann T, van Wijk N, Wurtman RJ, Olde Rikkert MG, Sijben JW, Soininen $\mathrm{H}$, et al. A nutritional approach to ameliorate altered phospholipid metabolism in Alzheimer's disease. J Alzheimers Dis. 2014; 41(3): 715-717.

5. Huang Y, Li W, Su ZY, Kong AN. The complexity of the Nrf2 pathway: Beyond the antioxidant response. The Journal of Nutritional Biochemistry. 2015; 26(12): 1401-1413.

6. Loboda A, Damulewicz M, Pyza E, Jozkowicz A, Dulak J. Role of Nrf2/HO-1 system in development, oxidative stress response and diseases: an evolutionarily conserved mechanism. Cellular and Molecular Life Sciences. 2016; 73(17):3221-3247.

7. Konrad FM, Knausberg U, Höne R, Ngamsri KC, Reutershan J. Tissue heme oxygenase-1 exerts antiinflammatory effects on LPS-induced pulmonary inflammation. Mucosal Immunology. 2015; 9(1): 98-111.

8. Wang LL, Yu QL, Han L, Ma XL, Song RD, Zhao SN, et al. Study on the effect of reactive oxygen species-mediated oxidative stress on the activation of mitochondrial apoptosis and the tenderness of yak meat. Food Chemistry. 2018; 244:394-402. 
9. Piantadosi CA, Carraway MS, Babiker A, Suliman HB. Heme Oxygenase-1 Regulates Cardiac Mitochondrial Biogenesis via Nrf2-Mediated Transcriptional Control of Nuclear Respiratory Factor-1. Circulation Research. 2008; 103(11):1232-1240.

10. Mylroie H, Dumont O, Bauer A, Thornton CC, Mackey J, Calay D, et al. PKC -CREB-Nrf2 signalling induces $\mathrm{HO}-1$ in the vascular endothelium and enhances resistance to inflammation and apoptosis. Cardiovascular Research. 2015; 106(3):509-519.

11. Cui Y, Ma S, Zhang C, Li D, Yang B, Lv P, et al. Pharmacological activation of the Nrf2 pathway by $3 \mathrm{H}-$ 1, 2-dithiole-3-thione is neuroprotective in a mouse model of Alzheimer disease. Behavioural Brain Research. 2018; 336:219-226.

12. Sun $M$, Zhang L, Song S, Dong Y, Zhang Y, Xie Z. Effect of sevoflurane on the expression of microglia inflammatory cytokines in cultured mice. Progress in Modern Biomedicine. 2015; 15(15): 2840-2844.

13. Kilkenny C, Browne WJ, Cuthill IC, Emerson M, Altman DG. Improving bioscience research reporting: the ARRIVE guidelines for reporting animal research. PLoS Biol. 2010; 8: e1000412.

14. Qiu L, Zhu C, Bodogan T, Gómez-Galán M, Zhang Y, Zhou K, et al. Acute and Long-Term Effects of Brief Sevoflurane Anesthesia During the Early Postnatal Period in Rats. Toxicol Sci. 2016; 149(1): 121-133.

15. Fan D, Li J, Zheng B, Hua L, Zuo Z. Enriched Environment Attenuates Surgery-Induced Impairment of Learning, Memory, and Neurogenesis Possibly by Preserving BDNF Expression. Mol Neurobiol. 2016; 53(1): 344-354.

16. Van de Rest O, Wang Y, Barnes LL, Tangney C, Bennett DA, Morris MC. APOE epsilon4 and the associations of seafood and long-chain omega-3 fatty acids with cognitive decline. Neurology. 2016; 86(22): 2063-2070.

17. Drobish JK, Gan ZS, Cornfeld AD, Eckenhoff MF. From the Cover: Volatile Anesthetics Transiently Disrupt Neuronal Development in Neonatal Rats. Toxicol Sci. 2016; 154(2): 309-319.

18. Zhao Y, Chen K, Shen X. Environmental Enrichment Attenuated Sevoflurane-Induced Neurotoxicity through the PPAR-gamma Signaling Pathway. Biomed Res Int. 2015; 2015: 107149.

19. Ho CF, Bon CP, Ng YK, Herr DR, Wu JS, Lin TN, et al. Expression of DHA-Metabolizing Enzyme Alox15 is Regulated by Selective Histone Acetylation in Neuroblastoma Cells. Neurochem Res. 2018; 43(3):540-555.

20. Valentini KJ, Pickens CA, Wiesinger JA, Tenton JI. The effect of fish oil supplementation on brain DHA and EPA content and fatty acid pro file in mice. Int J Food Sci Nutr. 2018; 69(6):705-717.

21. Chuang DY, Simonyi A, Kotzbauer PT, Gu Z, Sun GY. Cytosolic phospholi pase A2 plays a crucial role in ROS/NO signaling during microglialactivation through the lipoxygenase pathway. $J$ Neuroinflamma tion. 2015; 12: 199.

22. Shu L, Wang C, Wang J, Zhang Y, Zhang X, Yang Y. The neuroprotection of hypoxic preconditioning on rat brain against traumatic brain injury by up-regulated transcription factor Nrf2 and HO-1 expression. Neuroscience Letters. 2016; 611:74-80. 
23. Wang Z, Zhang H, Sun X, Ren L. The protective role of vitamin D3 in a murine model of asthma via the suppression of TGF- $\beta / \mathrm{Smad}$ signaling and activation of the Nrf2/HO-1 pathway. Molecular Medicine Reports. 2016; 14(3):2389-2396.

\section{Tables}

Table 1 Escape latency period of the rats at different time points. $(n=9, \pm S)$

\begin{tabular}{|llllll|}
\hline Groups & Day $1(\mathrm{sec})$ & Day $2(\mathrm{sec})$ & Day 3 $(\mathrm{sec})$ & Day $4(\mathrm{sec})$ & Day $5(\mathrm{sec})$ \\
\hline Control & $58.65 \pm 4.07$ & $47.68 \pm 3.69$ & $38.81 \pm 3.35$ & $30.61 \pm 2.65$ & $19.52 \pm 1.44$ \\
\hline Model & $82.29 \pm 7.53^{\star}$ & $70.56 \pm 5.31^{\star}$ & $59.39 \pm 3.78^{\star}$ & $43.89 \pm 1.45^{\star}$ & $38.56 \pm 2.41^{\star}$ \\
\hline L-DHA & $75.69 \pm 2.99^{\star}$ & $67.01 \pm 2.12^{\star}$ & $53.18 \pm 2.56^{\star}$ & $40.45 \pm 3.20^{\star}$ & $32.72 \pm 2.24^{\star}$ \\
\hline M-DHA & $69.19 \pm 2.22^{\star}$ & $58.84 \pm 3.54^{\star} \triangle$ & $48.98 \pm 2.12^{\star} \triangle$ & $34.59 \pm 2.12^{\star} \triangle$ & $26.85 \pm 1.12^{\star} \triangle$ \\
\hline H-DHA & $61.57 \pm 2.78 \triangle$ & $53.22 \pm 1.91 \triangle$ & $40.62 \pm 1.72 \triangle$ & $32.41 \pm 2.21 \triangle$ & $20.01 \pm 2.07 \triangle$ \\
\hline
\end{tabular}

Compared with Control group, ${ }^{*} \mathrm{P}<0.05$; compared with Model group, $\triangle \mathrm{P}<0.05$

Table 2 Comparison of Learning Ability Test Results in Each Group $(n=9, \pm S)$

\begin{tabular}{|lll|}
\hline Groups & Number of Times to Cross the Platform & Time for Target Quadrant Stay $(\mathrm{sec})$ \\
\hline Control & $8.63 \pm 1.07$ & $67.68 \pm 13.69$ \\
\hline Model & $4.96 \pm 0.63^{\star}$ & $42.56 \pm 9.31^{\star}$ \\
\hline L-DHA & $6.63 \pm 0.79 *$ & $57.08 \pm 11.12^{\star}$ \\
\hline M-DHA & $7.29 \pm 0.52$ & $60.84 \pm 13.54 \triangle$ \\
\hline H-DHA & $8.17 \pm 0.38^{\triangle}$ & $63.97 \pm 11.91^{\triangle}$ \\
\hline
\end{tabular}

Compared with Control group, ${ }^{*} \mathrm{P}<0.05$; compared with Model group, $\triangle \mathrm{P}<0.05$

\section{Figures}


A
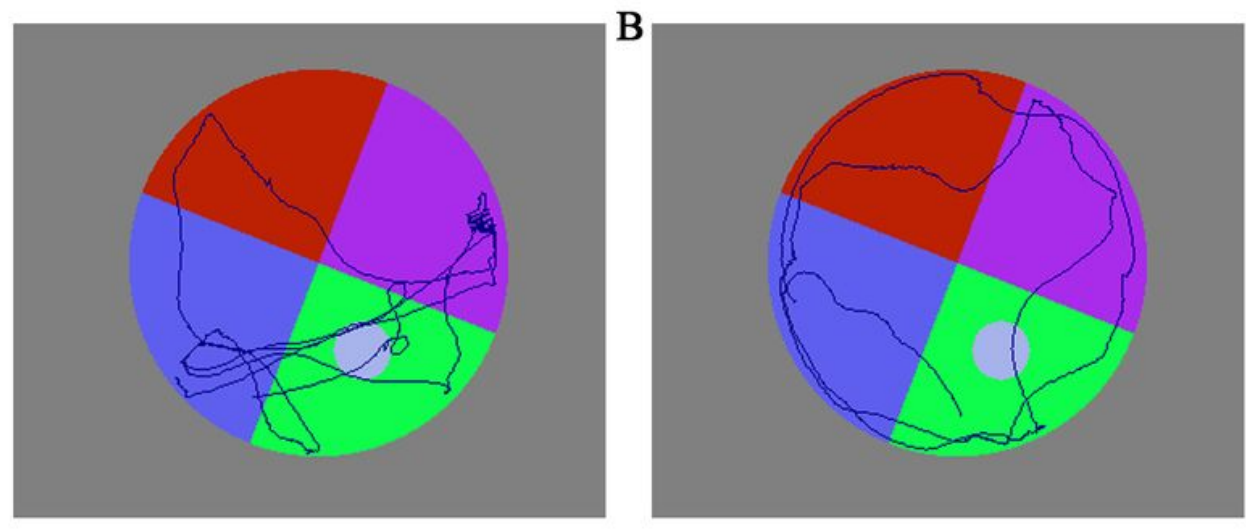

$\mathrm{C}$
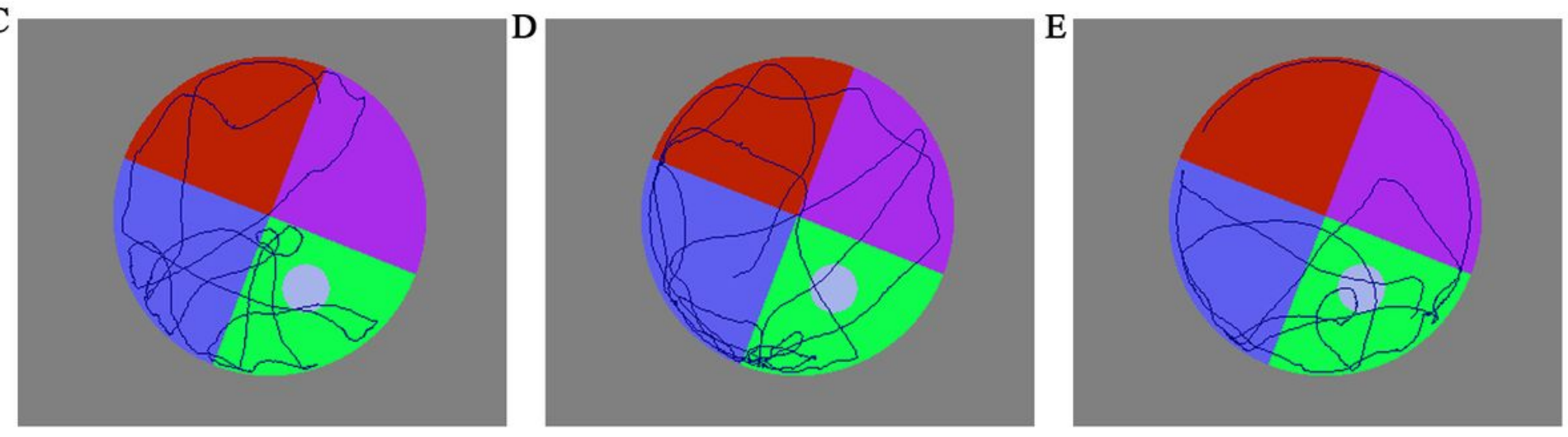

\section{Figure 1}

The swimming trajectory of each group. (A) Control group. (B) Model group. (C) L-DHA group. (D) M-DHA group. (E) H-DHA group. DHA, Docosahexaenoic acid; L, low-dose; $\mathrm{M}$, medium-dose; H, high-dose.

A

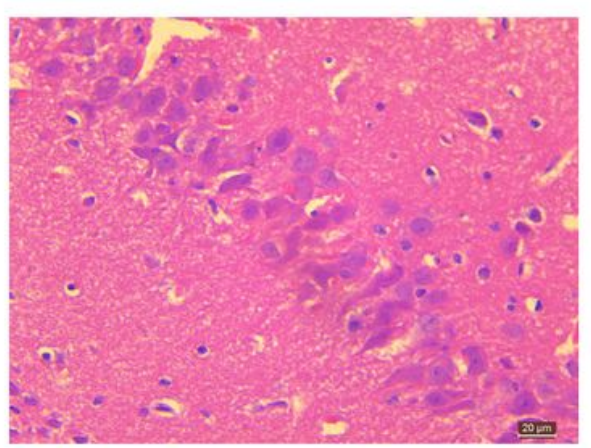

$\mathbf{C}$

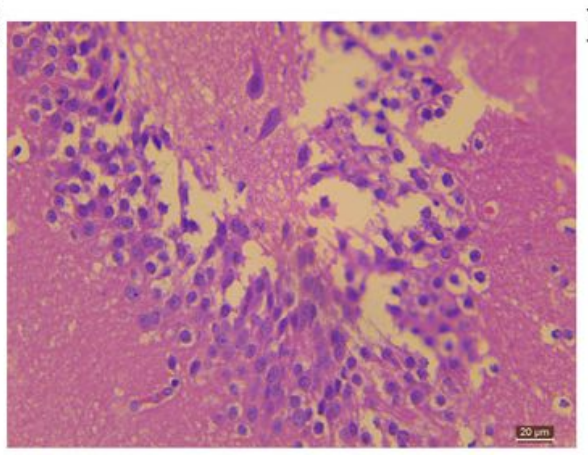

B
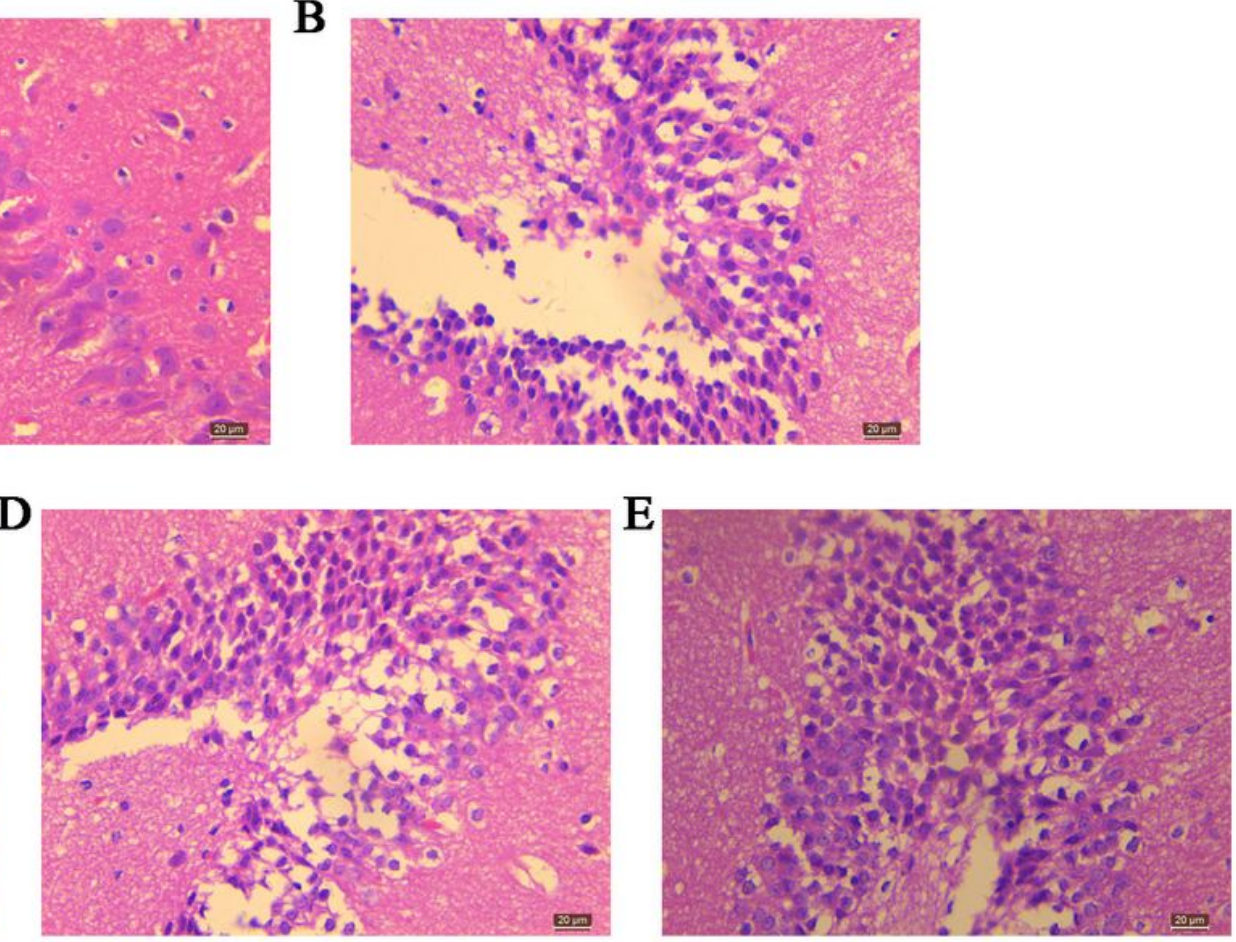
Figure 2

The pathological changes in the hippocampus measured using haematoxylin and eosin staining. The magnification was $x 400$; scale bar, $20 \mu \mathrm{m}$. (A) Control group. (B) Model group. (C) L-DHA group. (D) MDHA group. (E) H-DHA group. DHA, Docosahexaenoic acid; L, low-dose; M, medium-dose; H, high-dose.

A
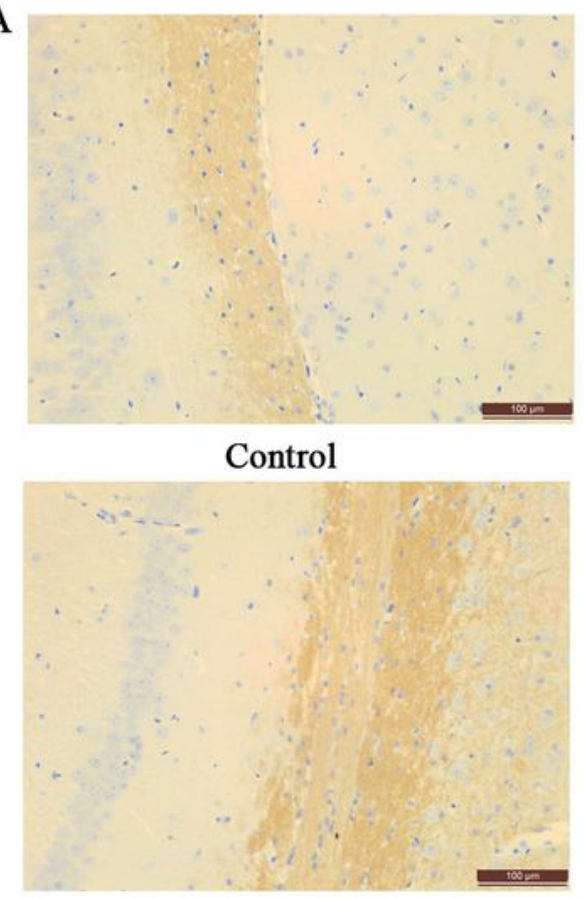

M-DHA

C

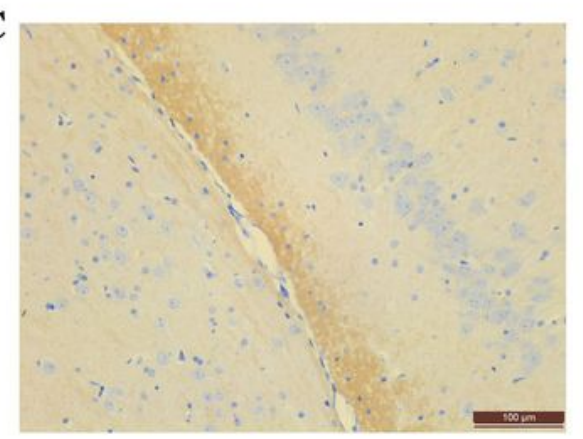

Control

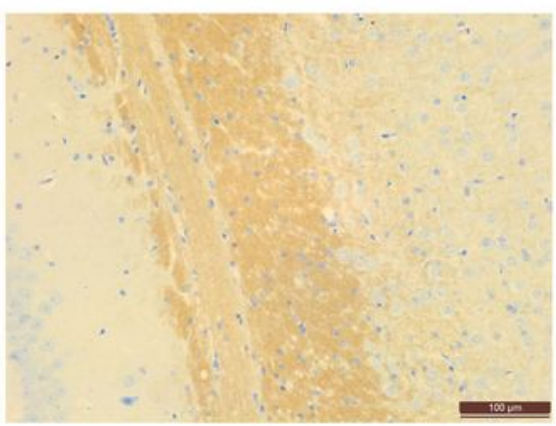

M-DHA

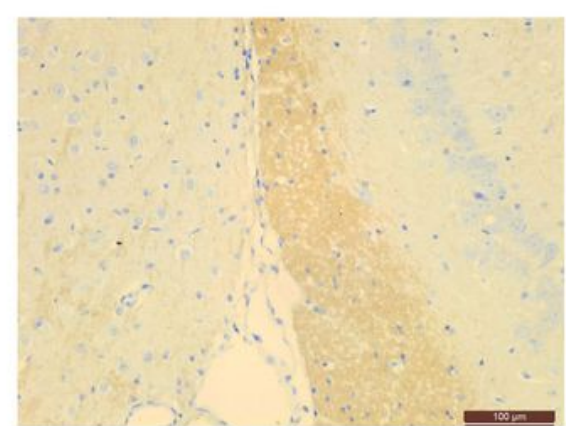

Model

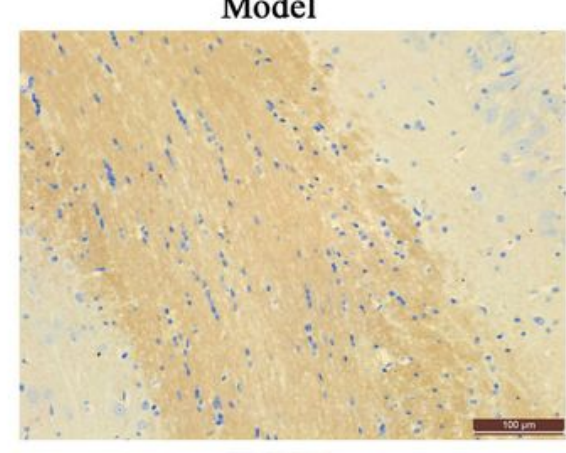

H-DHA

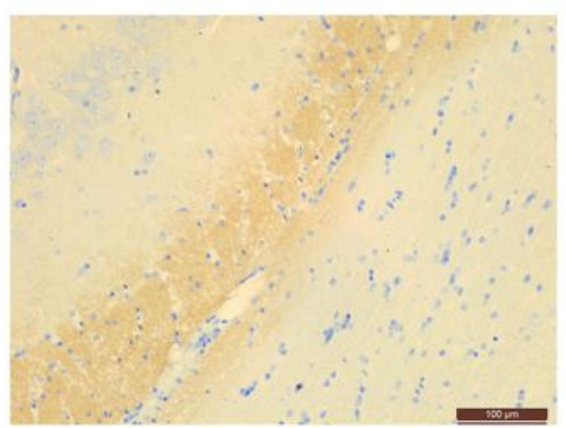

Model

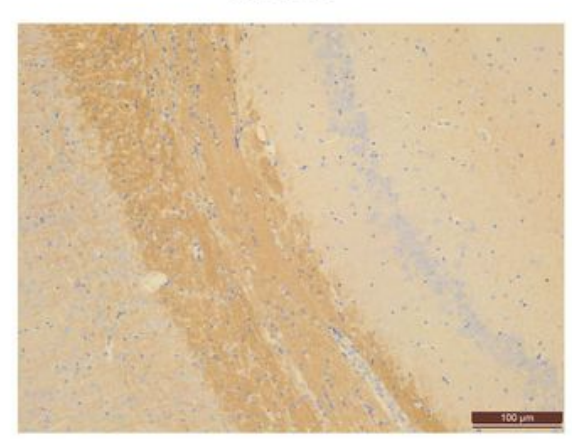

H-DHA
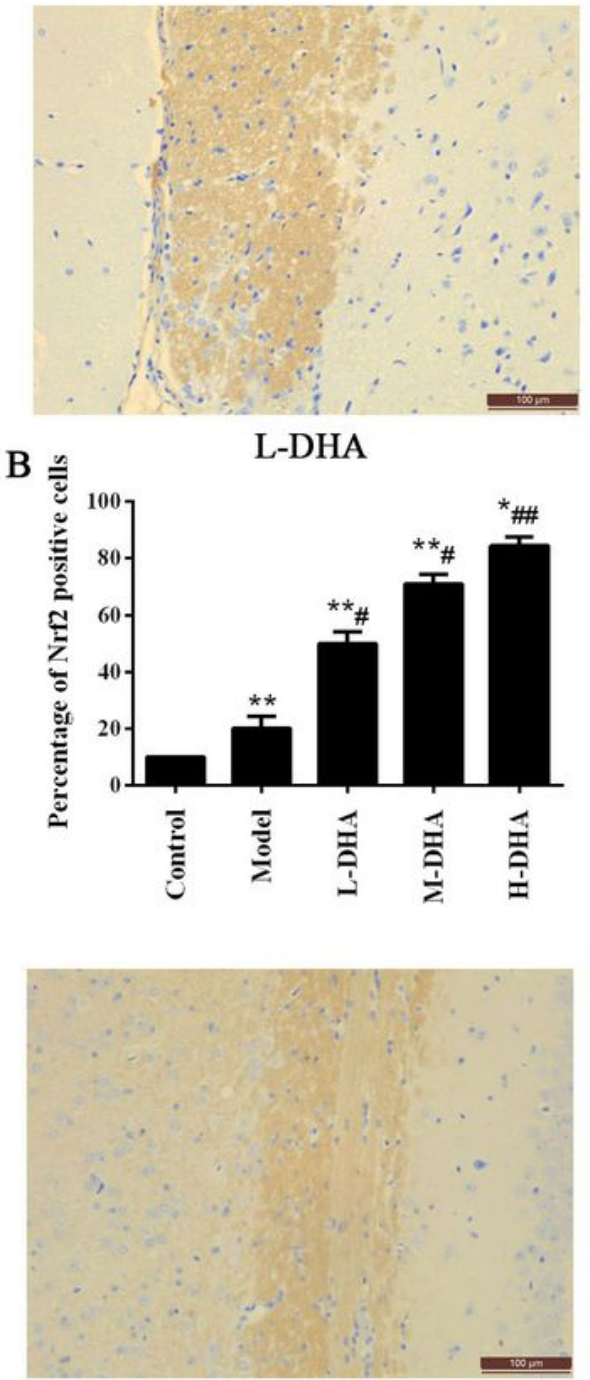

L-DHA

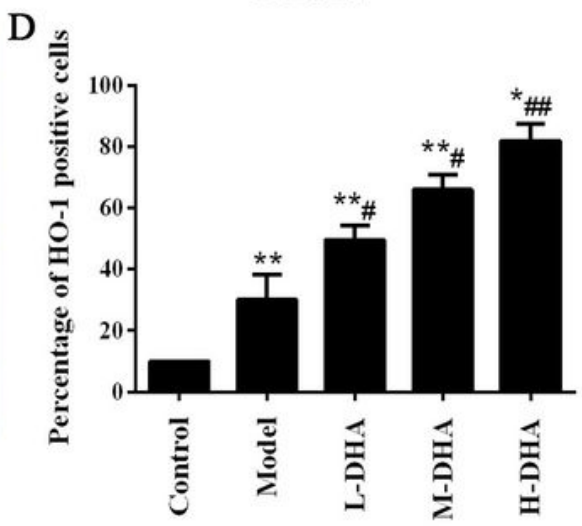

Figure 3 
The expression of Nrf2 and HO-1 detected by immunohistochemistry. The magnification was x200; scale bar, $100 \mu \mathrm{m}$. (A) The expression of Nrf2 protein. (B) Percentage of Nrf2 positive cells from each group. (C) The expression of HO-1 protein. (D) Percentage of HO-1 positive cells from each group. ${ }^{*} \mathrm{P}<0.05$ vs. Control group, ${ }^{\star *} \mathrm{P}<0.01$ vs. Control group; $\# \mathrm{P}<0.05$ vs. Model group, $\# \# \mathrm{P}<0.01$ vs. Model group.

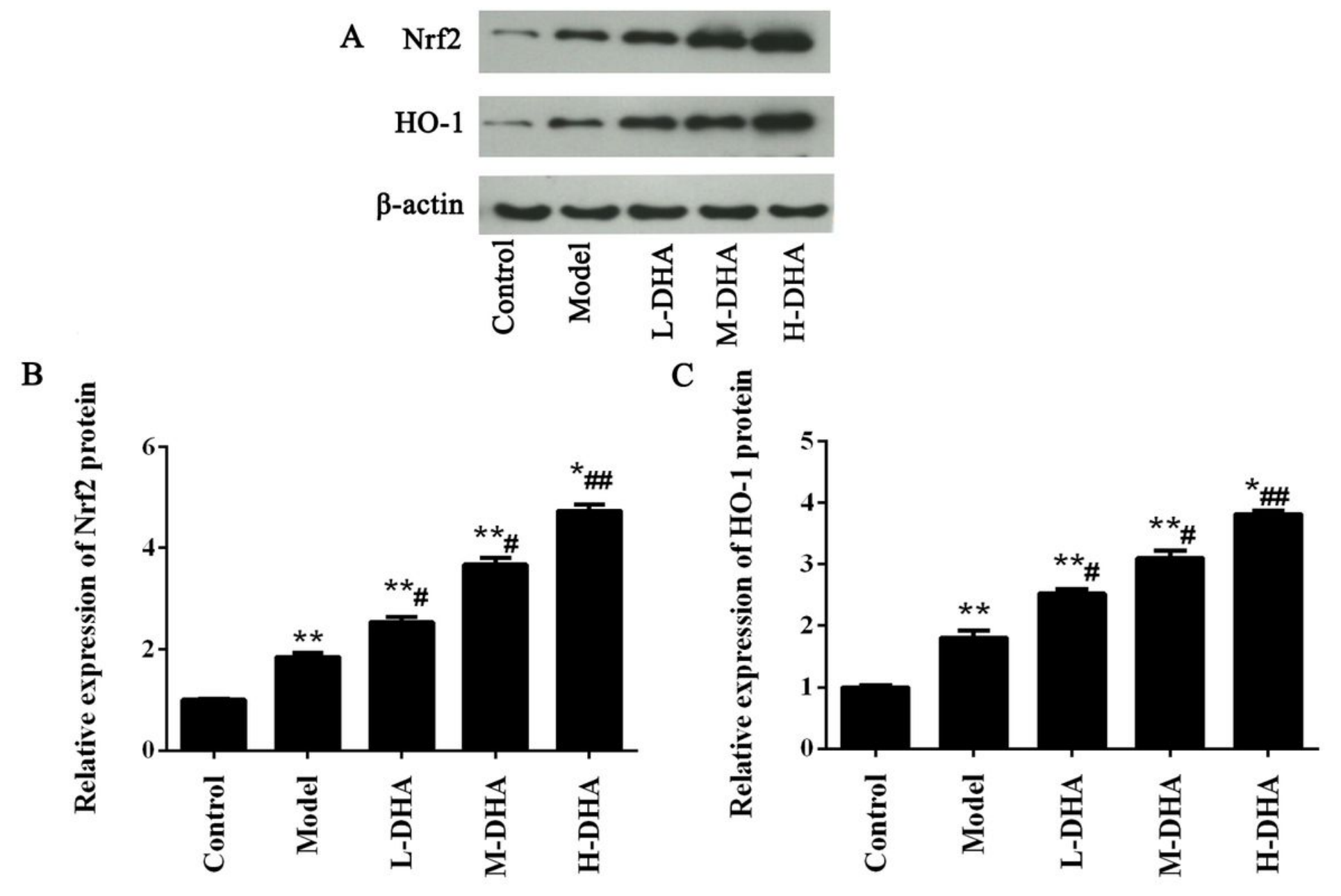

Figure 4

Nrf2 and HO-1 expression. (A) Western blot analysis was performed to determine the Nrf2 and HO-1 protein level in each group. (B) Quantification of the Nrf2 protein expression. (C) Quantification of the HO1 protein expression. ${ }^{*}<<0.05$ vs. Control group, ${ }^{\star \star P} P<0.01$ vs. Control group; $\# \mathrm{P}<0.05$ vs. Model group, $\# \# \mathrm{P}<0.01$ vs. Model group.

\section{Supplementary Files}

This is a list of supplementary files associated with this preprint. Click to download.

- NC3RsARRIVEGuidelinesChecklist2014.pdf

- Nrf2.bmp 
- H01.bmp

- renameda5bcb.bmp

Page 15/15 